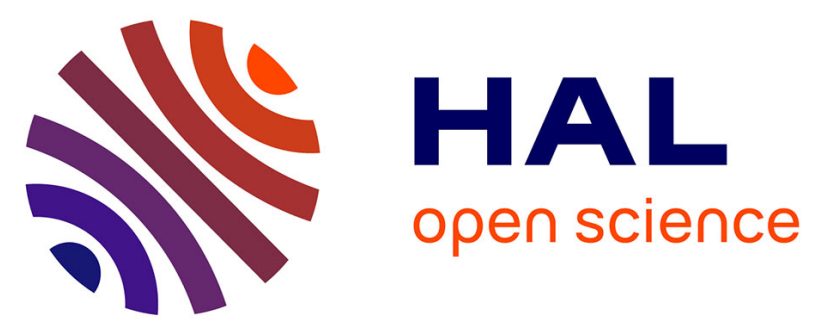

\title{
Isotropically coercive free layer integration in a magnetic tunnel junction for neuromorphic applications
}

Marco Mansueto, Antoine Chavent, Ricardo C. Sousa, Liliana D. Buda-Prejbeanu, Ioan Prejbeanu, Bernard Diény

\section{- To cite this version:}

Marco Mansueto, Antoine Chavent, Ricardo C. Sousa, Liliana D. Buda-Prejbeanu, Ioan Prejbeanu, et al.. Isotropically coercive free layer integration in a magnetic tunnel junction for neuromorphic applications. 2020 IEEE International Memory Workshop (IMW), May 2020, Dresden, Germany. pp.1-4, 10.1109/IMW48823.2020.9108129 . hal-03111548

\author{
HAL Id: hal-03111548 \\ https://hal.science/hal-03111548
}

Submitted on 1 Sep 2021

HAL is a multi-disciplinary open access archive for the deposit and dissemination of scientific research documents, whether they are published or not. The documents may come from teaching and research institutions in France or abroad, or from public or private research centers.
L'archive ouverte pluridisciplinaire HAL, est destinée au dépôt et à la diffusion de documents scientifiques de niveau recherche, publiés ou non, émanant des établissements d'enseignement et de recherche français ou étrangers, des laboratoires publics ou privés.

\section{(c)(1)}

Distributed under a Creative Commons Attribution| 4.0 International License 


\section{Isotropically coercive free layer integration in a magnetic tunnel junction for neuromorphic applications}

\author{
Marco Mansueto \\ Univ. Grenoble Alpes, CEA, CNRS, \\ Grenoble INP \\ SPINTEC \\ Grenoble, France \\ marco.mansueto@cea.fr
}

Liliana D. Buda-Prejbeanu

Univ. Grenoble Alpes, CEA, CNRS,

Grenoble INP

SPINTEC

Grenoble, France

liliana.buda@cea.fr

\author{
Antoine Chavent \\ Univ. Grenoble Alpes, CEA, CNRS, \\ Grenoble INP \\ SPINTEC \\ Grenoble, France \\ antoine.chavent@cea.fr \\ Ioan L. Prejbeanu \\ Univ. Grenoble Alpes, CEA, CNRS, \\ Grenoble INP \\ SPINTEC \\ Grenoble, France \\ ioan.prejbeanu@cea.fr
}

\author{
Ricardo Sousa \\ Univ. Grenoble Alpes, CEA, CNRS, \\ Grenoble INP \\ SPINTEC \\ Grenoble, France \\ ricardo.sousa@cea.fr \\ Bernard Dieny \\ Univ. Grenoble Alpes, CEA, CNRS, \\ Grenoble INP \\ SPINTEC \\ Grenoble, France \\ bernard.dieny@cea.fr
}

\begin{abstract}
The concept of a spintronic memristor based on angular variation of Tunnel Magnetoresistance relies on the development of an isotropically coercive free layer able to stabilize the magnetization in any in-plane direction. In this work, we first demonstrate those properties in a composite ferromagnet/antiferromagnet/ferromagnet trilayer by performing hysteresis loops at different in-plane angles and rotating field experiments. In the second part, we describe the material composition of the total stack and the fabrication process to create the final device. In the last part, the hysteresis loops at different in-plane angles and the rotating field experiments are performed at device level and compared with the ones performed at full sheet film, confirming the magnetically isotropic nature of the free layer.
\end{abstract}

Keywords—spintronics, memristor, MTJ, TMR

\section{INTRODUCTION}

The exponentially increasing amount of data in the information and communication technology (ICT) world in the last decade results in a corresponding increase of electrical power consumption associated to it. In 2018, it was estimated that the ICT's amount of data generated worldwide reached 20 Zettabytes (20 bilion Terabytes). The power consumption associated to ICT is estimated to represent about $10 \%$ of the worldwide total electrical consumption [1]. The main causes in ICT power consumption are associated with the wireless transmission of data, displays, and inefficient computer architectures. To address this issue of ICT power consumption, the scientific community is looking to the most efficient and lowest power consuming system known, the human brain. The concept of AI is born to try to emulate the complex functionalities of the brain. Between those, the development of an architecture with massive parallelization of processing units (neurons) and memory units (synapses) seems to be a promising solution. In the last few years, several devices were proposed to mimic both neuron and synapse working principles. For the specific case of synapses, the concept of memristor, a device with a resistance value dependent on the history of the electrical charges passed through it, developed in the 70's [2], has been exploited for the realization of devices using different technologies. An example is a device that is able to change resistance value thanks to the voltage dependent ion migration (as for example oxygen ions) in materials like $\mathrm{Ti}_{\mathrm{x}} \mathrm{O}_{1-\mathrm{x}}$ or $\mathrm{Hf}_{\mathrm{x}} \mathrm{O}_{1-\mathrm{x}}$ [3], [4]. In this particular case, problems of endurance represent the main limitation since learning processes usually involve a large number of write cycles. In contrast, spintronic memristors do not present this problem, since the information relies on the magnetization state and no atomic motion takes place during the writing process. An example of this is a magnetic tunnel junction (MTJ) with a domain wall in the free layer that can be moved through current pulses, thus varying the proportion of MTJ area in parallel and anti-parallel configuration and correlatively reaching different resistance states [5]. Another example exploits spin-orbit torque (SOT) in an antiferromagnetic material exchange biased with an out-ofplane magnetized ferromagnet that is gradually switched [6], [7]. A third example uses a chain of MTJs in serial configuration, in which the MTJ can be switched one by one, thus achieving a gradual change in the total resistance [8]. However, a drawback of these above mentioned spintronic devices is their relatively large size (micronic dimension) required to get several intermediate resistance states.

The device we propose in this work is based on a single nanosized pillar very similar to a usual MRAM cell. The idea to achieve several resistance states is to exploit the dependence of the tunnel magnetoresistance (TMR) on the angle between the magnetization of the two ferromagnetic electrodes in an in-plane MTJ (the analyzer layer AL and the free layer FL in Fig. 1a) [9]. The lowest resistance value is obtained in the case of parallel configuration of the two magnetization while the antiparallel case gives the highest resistance. In between the two, the TMR is varying with the cosine of the angle as shown in Fig. 1b. The writing mechanism relies on the spin-transfer torque (STT) created by an out-of-plane spin polarized current injected in the FL layer. This polarized current is obtained thanks to an additional out-of-plane magnetized polarizer (POL) placed underneath the FL. This current generates an out-of-plane precession of the FL magnetization (shown as dashed line in Fig. 1a). The application of current pulses rather than a DC current enables clockwise or anticlockwise step by step discrete rotations of the FL magnetization achieving 
intermediate resistance states. The use of an additional inplane field perpendicular to the AL magnetization direction allows limiting the angular excursion of the FL magnetization to $\pm 90^{\circ}$ around this transverse field thus insuring the monotonicity of the resistance variation with the polarity of the applied voltage as required for a memristive behavior. A more accurate description of the functionalities of the device is given in [10].

In this work, we present how to realize a FL able to stabilize the magnetization along different in-plane directions with isotropic thermal stability by creating an isotropically coercive free layer and how to integrate it in a complex structure as in Fig. 1a. After a nano-fabrication process, magnetic characterizations were performed to confirm the magnetically isotropic nature of the free layer.
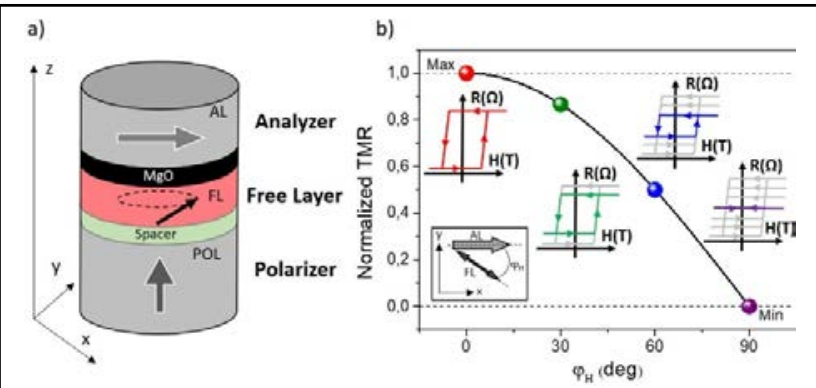

Fig. 1 a) Schematics of the device. b) TMR variation with the in-plane angle between analyzer and free layer as in the inset and schematics of the relative resistance loops.

\section{ISOTROPICALLY COERCIVE FREE LAYER}

\section{A. Realization}

Isotropic magnetic properties were already observed in amorphous in-plane magnetized ferromagnetic systems in which the disordered crystalline structure creates a distribution of anisotropy directions all over the plane [11]. In this work, instead, we exploit a structure based on the coupling between ferromagnet FM and thin antiferromagnet AF. It is well-known that, above a certain thickness of the AF layer, its anisotropic energy is strong enough to pin the FM magnetization along an in-plane direction thanks to the exchange bias phenomenon. However, for lower thicknesses, this AF pinning becomes too weak and the AF spin lattice gets entirely dragged due to the interfacial F/AF coupling when the F magnetization rotates or switches. This results in an increase in $\mathrm{F}$ coercive field while the exchange bias field is still negligible. In such system, similarly to the random anisotropy materials, the dissipation is not associated to the presence of a well-defined anisotropy axis but to numerous magnetization rearrangements at the nanometer scale occurring during the magnetization evolution. As a result, this dissipative mechanism is isotropic. From conceptual point of view, it can be described by a dry friction-like term which can be introduced in the Landau Lifshitz Gilbert equation (for more details [10]).

\section{B. Vibrating Sample Magnetometer Characterization}

To determine the right AF thickness yielding this isotropically coercive behavior, a study of exchange bias and coercive field dependence on AF thickness was performed on a trilayer sample made of unpatterned $\mathrm{Py}(1 \mathrm{~nm}) / \mathrm{IrMn}(\mathrm{x}) / \mathrm{Py}(1 \mathrm{~nm})$. The sample was deposited by magnetron sputtering and annealed at $300^{\circ} \mathrm{C}$ for $1 \mathrm{~h} 30$ under an in-plane field of $230 \mathrm{mT}$. In Fig. $2 \mathrm{a}$, it is shown that, for IrMn thicknesses around $2.1 \mathrm{~nm}$, while the exchange bias is still negligible, a steep increase of coercive field is observed as expected. This particular IrMn thickness was chosen for the following experiments.
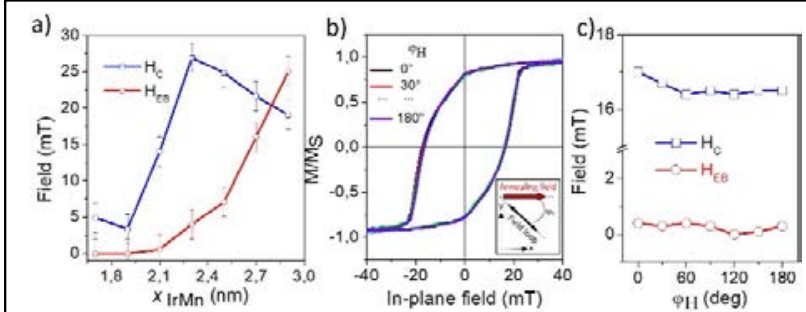

Fig. 2 a) Coercive field $\mathrm{H}_{C}$ and exchange bias $\mathrm{H}_{\mathrm{EB}}$ dependence on IrMn thickness. b) Hysteresis loops at different in-plane angles with respect to the annealing field direction (red arrow in the inset). c) Coercive field $\mathrm{H}_{\mathrm{C}}$ and exchange bias $\mathrm{H}_{\mathrm{EB}}$ dependence on the in-plane angle $\varphi_{\mathrm{H}}$. [10]

Measurements at different in-plane angles (Fig. 2b) show that the magnetic properties such as coercive field and remanence are almost independent on this angle as in Fig. 2c, confirming the isotropic nature of the free layer. Note that the rounded shape of the loop is expected as a result of dry friction torque due to the F/AF coupling (see [10]).

\section{Rotating Field Characterization}

The same sample was used for a rotating field experiment using a planar Hall (PHR) measurement setup [12].
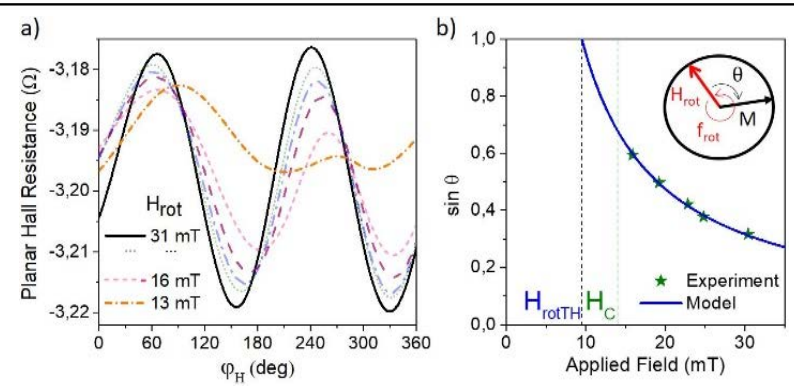

Fig. 3 a) Planar Hall resistance (PHR) under rotating field of frequency of $10 \mathrm{~Hz}$ and different amplitudes. b) Angular shift dependence on field amplitude with dry friction model (blue line) and experimentally obtained (green stars). [10]

As expected, for large rotating field amplitude $\mathrm{H}_{\text {rot }}$, the resistance varies as $\sin \left(2 \varphi_{\mathrm{H}}\right)$ (black curve in Fig. 3a). For smaller amplitudes of the field, the curve gradually shifts creating a dragg angle between the rotating field and the magnetization. For fields smaller than the coercive field, the magnetization no more follows the rotating field (as in the $13 \mathrm{mT}$ curve of Fig. 3a). The observed gradual increase of the amplitude PHR signal versus $\mathrm{H}_{\text {rot }}$ amplitude is also expected since the amount of spins following the field increases as $\mathrm{H}_{\text {rot }}$ amplitude increases. Fig. 3b shows the phase shifts between magnetization and field (i.e the dragg angle) versus field amplitude. This variation can be well fitted by the analytical solution obtained with the dry friction model. This is a further confirmation of the isotropic properties of the unpatterned free layer. 


\section{INTEGRATION IN THE FULL STACK AND FABRICATION}

Here we briefly describe the material stack used for the device as in Fig. 1a and the fabrication process to pattern such device. All the thicknesses in parenthesis are in $\mathrm{nm}$.

\section{A. Full Stack}

The F/AF/F trilayer constituting the device FL was inserted in the full structure as represented in Fig. 4a. The samples were deposited by magnetron sputtering at Spintec. After a seed layer of $\mathrm{Pt}$, an out-of-plane synthetic antiferromagnet (SAF) was deposited by exploiting the perpendicular anisotropy of a multilayer structure of [Co $(0.5) / \mathrm{Pt}(0.25)]_{6}$ and $[\mathrm{Co}(0.5) / \mathrm{Pt}(0.25)]_{3}$ separated by a thin layer of $\mathrm{Ru}(0.9)$ to exploit the peak of the antiferromagnetic RKKY coupling. The SAF is coupled to a polarizer layer of $\mathrm{FeCoB}(1.2)$ in contact with the first $\mathrm{MgO}$ barrier with a relatively low resistance*area product of $2 \Omega \mu \mathrm{m}^{2}$. The F/AF/F trilayer structure was then inserted between two $\mathrm{FeCoB}(1.6)$ layers in contact with the top and bottom $\mathrm{MgO}$ barrier. The second $\mathrm{MgO}$ barrier with a resistance*area product of $10 \Omega \mu \mathrm{m}^{2}$ was optimized to give a TMR as large as possible between the free layer and an in-plane magnetized synthetic antiferromagnet placed above the top tunnel barrier. This pinned top analyzer layer has the composition: $\mathrm{FeCoB}(1.2) / \mathrm{Ta}(0.2) / \mathrm{Co}(1) / \mathrm{Ru}(0.9) / \mathrm{Co}(2.5) / \operatorname{IrMn}(7) . \quad \mathrm{A}$ capping layer of $\mathrm{Cu}$ and $\mathrm{Pt}$ protects the stack from oxidation and constitutes the top electrical contact to the stack.

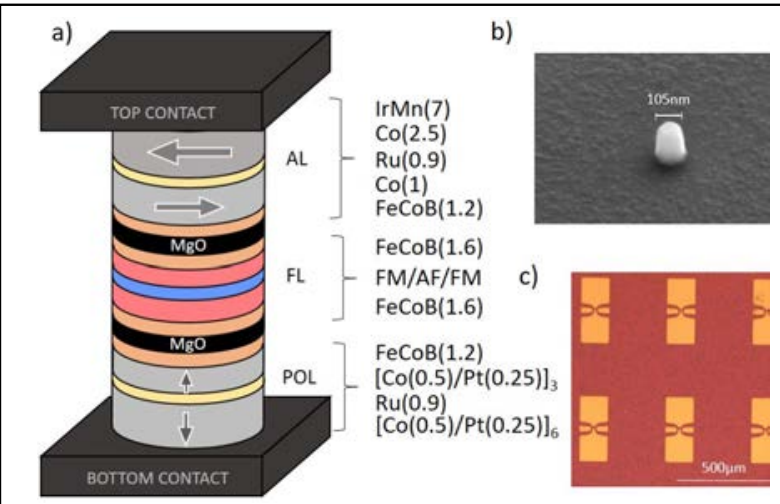

Fig. 4 a) Schematics of the device material stack with thicknesses in nm. b) SEM image of a hard mask after RIE etching. c) Top view of the two contacts.

\section{B. Nano-Fabrication Process}

The samples were annealed at $300^{\circ} \mathrm{C}$ for $1 \mathrm{~h} 30$ under an in-plane magnetic field of $230 \mathrm{mT}$. The field is intended to set the orientation of the in-plane analyzer magnetization. After deposition of an hard mask of Ta, e-beam lithography was used to create circular resist patterns with diameters between $20 \mathrm{~nm}$ and $150 \mathrm{~nm}$. A Reactive Ion Etching machine was then used to etch the Ta layer and creates the hard mask Ta pillars (as in Fig. 4b). The magnetic stack was then etched by Ion Beam Etching at different incident angles. The etched stacks are then embedded in a planarizing resist which is subsequently partially etched to open the top electrical contacts. The contacts are then patterned as in Fig. 4c.

\section{CHARACTERIZATION RESUlts}

Magnetic characterizations were performed on these samples to test the success of the fabrication process and to assess the isotropic properties of the FL at device level. An example of resistance - field loop is presented in Fig. 5a. The device has a diameter of $80 \mathrm{~nm}$ and a total resistance consistent with the expected resistance*area product $\left(2+10 \Omega \mu \mathrm{m}^{2}\right)$. The TMR value is around $30 \%$ and the rounded shape of the loop is similar to the full sheet film one of Fig. 2b confirming the dry friction like reversal with field.

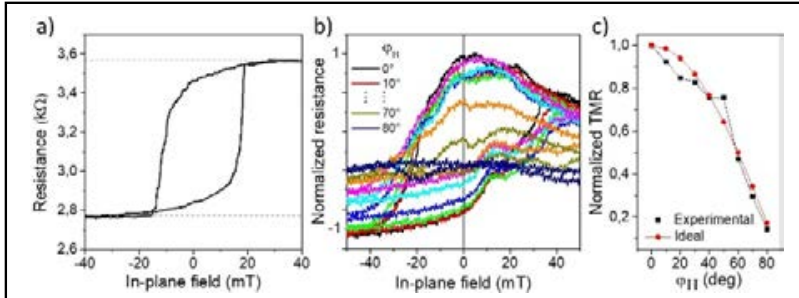

Fig. 5 a) Example of resistance loop of a device with $80 \mathrm{~nm}$ diameter. b) Example of evolution of resistance loop with the in-plane field angle. c) TMR variation with the in-plane angle in an ideal case (red line) and experimentally obtained.

\section{A. In-plane Field Loops}

A series of hysteresis loops measurement was performed at different in-plane angles with respect to the AL layer magnetization direction. This was realized with two orthogonal couples of coils with the sample placed at their center. The two components of the field (along x-axis and $y$ axis) were then adjusted to obtain different in-plane angles of the field. As shown in Fig. 5b, the coercive field of the different loops do not vary while the high and low resistance states at zero field are gradually shifting towards the center. This demonstrates that the magnetic properties are again independent on the in-plane angle even at device level. The obtained variation of TMR was directly compared to the cosine variation expected in the ideal case in Fig. 5c showing good agreement.

\section{B. Rotating Field Experiment}

Rotating field experiments were performed on a similar setup as the previous one. The two couples of coils along $\mathrm{x}$ and $y$ directions are supplied by $90^{\circ}$ out-of-phase current creating a rotating field on the device of constant amplitude. The rotating frequency could be varied up to $5 \mathrm{~Hz}$. The results shown in Fig. 6a correspond to an applied field of 40mT and frequency of $2 \mathrm{~Hz}$. The resistance (Fig. 6b) is oscillating between its maximum and its minimum value with the same frequency as the rotating field one. Note that the resistance smoothly varies in quasi-sine form, passing through all the intermediate values. In contrast to this, if the same experiment is performed on a standard binary MTJ, the result of Fig. $6 c$ is observed. The resistance values are then distributed non uniformly with two main regions in the high resistance region and in the low resistance region. The magnetization does not follow smoothly the rotating field. Due to its uniaxial anisotropy, during its rotation, the FL magnetization undergoes irreversible jumps while it crosses its hard axis direction. Also in this case, the isotropic properties of the device can be confirmed. 


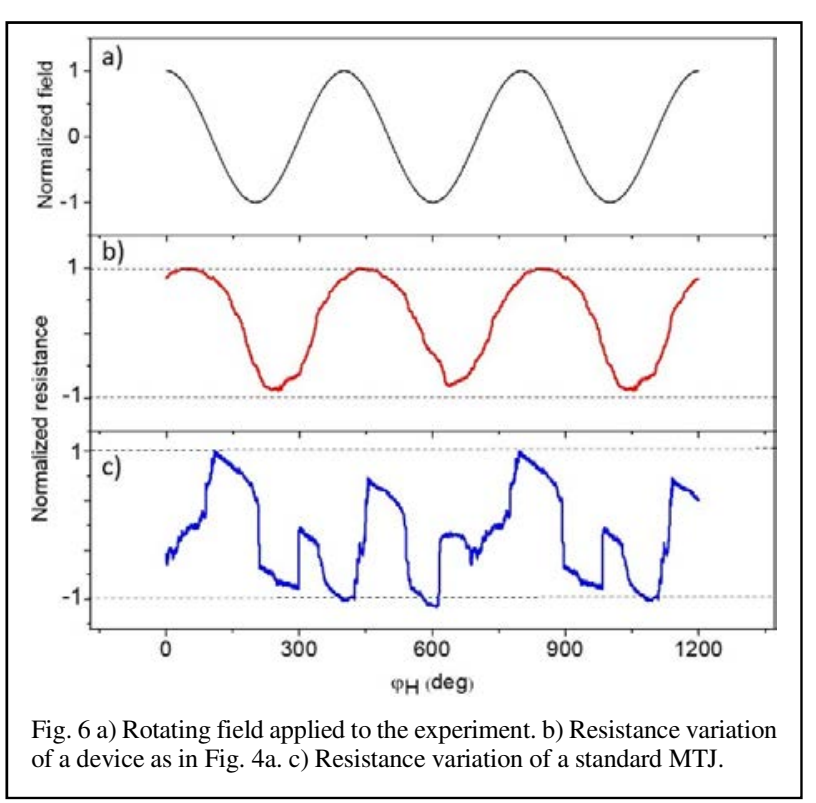

\section{CONCLUSION AND PERSPECTIVES}

In conclusion, a memristive device based on the angular variation of TMR requires the development of an isotropically coercive free layer able to stabilize the magnetization along different in-plane angles with equal thermal stability. In this study, we demonstrated the realization of such layer by exploiting the coupling at the FM/AF interface of a trilayer FM/AF/FM. The full sheet film measurements of coercivity and exchange bias at different inplane angles and the rotating field experiments confirmed the isotropic properties of the free layer. After the development of the total stack, and fabrication process, devices with diameter between $20 \mathrm{~nm}$ and $150 \mathrm{~nm}$ were measured with the same techniques. The hysteresis loops at different angles showed that the angular variation of TMR is, as expected, cosine-like while the coercive field is kept constant. The rotating field experiments demonstrated that the resistance can take all the intermediate values between its maximum and its minimum without any preference. This does not occur in a standard MTJ where the magnetization is stable along two opposite directions giving rise to a bimodal distribution of resistance values.

These promising results bring us to the next step where the use of Spin Transfer Torque through the application of pulses will allow the discrete rotation of the magnetization. The realization of this device would represent a good option for a scalable, easily integrable and low power consumption device for neuromorphic computing.

\section{ACKNOWLEDGMENT}

The authors want to acknowledge the Plateforme de Technologie Amont PTA for technical help in the fabrication process. Funding from ERC Advanced grant MAGICAL 669204 is acknowledged.

\section{REFERENCES}

[1] D. Reinsel et al., IDC White Paper Data Age 2025, (2017)

[2] L. O. Chua, Memristors-the missing circuit element, IEEE Trans. Circuit Theory CT-18, 507 (1971)

[3] D. B. Strukov , G. S. Snider, D. R. Stewart \& R. S. Williams, The missing memristor found, Nature, Vol 453, (2008)

[4] Y. Li, Z.Wang, R. Midya, Q. Xia, and J. J. Yang, Review of memristor devices in neuromorphic computing: Materials sciences and device challenges, J. Phys. D: Appl. Phys. 51, 503002 (2018)

[5] S. Lequeux, J. Sampaio, V. Cros, K. Yakushiji, A. Fukushima, R. Matsumoto, H. Kubota, S. Yuasa, and J. Grollier, A magnetic synapse: Multilevel spin-torque memristor with perpendicular anisotropy, Sci. Rep. 6, 31510 (2016).

[6] S. Fukami, C. Zhang, S. DuttaGupta, and H. Ohno, Magnetization switching by spin-orbit torque in an antiferromagnet-ferromagnet bilayer system, Nat. Mater. 15, 535 (2016).

[7] A. Kurenkov, S. D. Gupta, C. Zhang, S. Fukami,Y. Horio, and H. Ohno, Artificial Neuron and Synapse Realized in an Antiferromagnet/Ferromagnet Heterostructure Using Dynamics of Spin-Orbit Torque Switching, Adv. Mater., 31, 1900636 (2019)

[8] E. Raymenants, A. Vaysset, D. Wan, M. Manfrini, O. Zografos, O. Bultynck, J. Doevenspeck, M. Heyns, I. P. Radu, and T. Devolder, Chain of magnetic tunnel junctions as a spintronic memristor, J. Appl. Phys. 124, 152116 (2018).

[9] J. C. Slonczewski, Conductance and exchange coupling of two ferromagnets separated by a tunneling barrier, Phys. Rev. B 39, 6995 (1989).

[10] M. Mansueto, A. Chavent, S. Auffret, I. Joumard, J. Nath, I.M. Miron, U. Ebels, R.C. Sousa, L.D. Buda-Prejbeanu, I.L. Prejbeanu, and B. Dieny, Realizing an Isotropically Coercive Magnetic Layer for Memristive Applications by Analogy to Dry Friction, Phys. Rev. Applied 12, 044029 (2019)

[11] B. Dieny, B. Barbara, G. Fillion, M. Maeder, and B. Michelutti, Hysteresis loop and torque experiments on a random anisotropy system, J. Phys. 48, 1741 (1987)

[12] F. Nguyen Van Dau, A. Schuh, J. R. Childress, and M. Sussiau, Magnetic sensors for nanotesla detection using planar Hall effect, Sens. Actuators A 53, 256 (1996) 